\title{
Abstract \\ Hydrothermal Degradation of Biobased Poly(butylene succinate)/Nanofibrillated Cellulose Composites ${ }^{\dagger}$
}

\author{
Olesja Starkova ${ }^{1, *} \mathbb{C}$, Oskars Platnieks ${ }^{2}$, Alisa Sabalina ${ }^{1}$ and Sergejs Gaidukovs ${ }^{2, *}$ \\ 1 Institute for Mechanics of Materials, University of Latvia, Jelgavas 3, LV-1004 Riga, Latvia; \\ alisasabalina@gmail.com \\ 2 Institute of Polymer Materials, Faculty of Materials Science and Applied Chemistry, Riga Technical University, \\ P. Valdena 3/7, LV-1048 Riga, Latvia; oskars.platnieks@rtu.lv \\ * Correspondence: olesja.starkova@lu.lv (O.S.); sergejs.gaidukovs@rtu.lv (S.G.) \\ + Presented at the First Corrosion and Materials Degradation Web Conference, 17-19 May 2021; \\ Available online: https:// cmdwc2021.sciforum.net/.
}

check for

updates

Citation: Starkova, O.; Platnieks, O.; Sabalina, A.; Gaidukovs, S.

Hydrothermal Degradation of

Biobased Poly(butylene succinate)/

Nanofibrillated Cellulose Composites.

Mater. Proc. 2021, 6, 2. https://

doi.org/10.3390/CMDWC2021-09981

Academic Editor: Luigi Calabrese

Published: 8 May 2021

Publisher's Note: MDPI stays neutral with regard to jurisdictional claims in published maps and institutional affiliations.

Copyright: (c) 2021 by the authors. Licensee MDPI, Basel, Switzerland. This article is an open access article distributed under the terms and conditions of the Creative Commons Attribution (CC BY) license (https:// creativecommons.org/licenses/by/ $4.0 /)$.

\begin{abstract}
Biobased polymers and composites have gained increased global attention due to their abundance, renewability, and biodegradability. Natural fillers such as cellulose-based fillers improve the mechanical properties of biopolymers, extending their application range, while maintaining the eco-friendly characteristics of the materials. Mowing towards engineering applications, requirements imposed on materials' durability with regard to their environmental impact and high performance is necessary. Variations of ambient humidity and temperature could essentially reduce the service lifetime of biobased polymer composites. This study is focused on the hydrothermal degradation of poly(butylene succinate) (PBS) filled with nanofibrillated cellulose (NFC) by up to $50 \mathrm{wt}$ \% aimed at identifying the most efficient PBS/NFC composition, while maintaining a reasonable balance between the reinforcement effect and accelerated degradation that is inherent for most natural fillers. Water absorption and its effect on the structure, thermal, mechanical, and thermomechanical properties were studied. High reinforcement and adhesion efficiency is obtained for PBS/NFC composites and their properties are reasonably retained after hydrothermal ageing. Their water absorption capacity and diffusivity increased significantly with the NFC content in PBS. The degradation of the mechanical properties occurs to a greater extent with an increased NFC content in the polymer matrix. PBS, filled with $20 \mathrm{wt}$ \% of NFC, is identified as the most efficient composition, for which the negative environmental degradation effects are counterbalanced by the positive reinforcement effect.
\end{abstract}

Keywords: biopolymer; cellulose nanofibrils; durability; water absorption; mechanical properties; ageing; adhesion efficiency; biodegradation; modelling

Supplementary Materials: The conference presentation file is available at https:/ /www.mdpi.com/ article/10.3390/CMDWC2021-09981/s1.

Institutional Review Board Statement: Not applicable.

Informed Consent Statement: Not applicable. 